\title{
Perceptual enhancement of tumor targets in chest X-ray images
}

\author{
ELIZABETH A. KRUPINSKI, CALVIN F. NODINE, and HAROLD L. KUNDEL \\ University of Pennsylvania, Philadelphia, Pennsylvania
}

\begin{abstract}
Under tachistoscopic viewing conditions, precuing the location of potential lung tumor targets in chest X-ray images was less effective than precuing followed by bounding the region of interest (ROI) with a circle directly on the image. Detection performance increased as the image was systematically masked so that its size approximated that of the circled ROI. When viewing time was extended to allow shifts in eye position, circling the ROI was found to restrict the dispersion of fixations and increase the accuracy of fixating the target tumor. When targets were placed outside the ROI, the circle inhibited their detection relative to detection of targets inside the circled region. These findings suggest that cuing by circling restricts target detection to the ROI, and by doing so reduces the interfering effects of outside distractors that compete with the target for attention.
\end{abstract}

In medical X-ray imaging, tumors of the lungs, breasts, and other major organs make up a major class of search and detection targets. The detection and recognition of these targets is especially difficult because of (1) the presence of numerous anatomical objects that also possess similar features (e.g., blood vessels on end in the lungs); and (2) camouflaging effects created by projection X-ray imaging techniques. Essentially, the observer is searching a two-dimensional, translucent picture of internal human anatomy created from the shadows of the absorption pattern of X-rays passing through the body to the film plate, and interpreting these patterns as three-dimensional objects. The magnitude of the difficulty of this particular search and detection task is evident when error rates in radiology are considered. Misses (false-negatives) of tumors, fractures, and other diagnostic findings can be as high as $30 \%$, and false-positive rates typically run at $10 \%-15 \%$ (Bassett, Manjikian, \& Gold, 1990; Muhm, Miller, Fontan, Sanderson, \& Uhlenhopp, 1983).

Numerous attempts have been made to improve the tumor detection performance of the radiologist by cuing or directing visual search to potential target-containing image areas (Berbaum, Franken, Dorfman, \& Barloon, 1988; Berbaum et al., 1986; Carmody, Nodine, \& Kundel, 1980; Parker et al., 1982; Swensson, Hessel, \& Herman, 1977, 1982). Surprisingly, cuing in which clinical history information (e.g., a fall on the wrist, the location of a pain, the possibility of a fracture) is used to direct attention has

This work was supported by Grant CA-32870 from the National Cancer Institute, USPHS. Part of the work contained herein was based on the doctoral dissertation of E.A.K., Department of Psychology, Temple University. We would like to thank Lawrence $C$. Toto, for developing the computer display and tachistoscopic programs, and Charles F. Reed, for advice and comments as chair of E.A.K.'s dissertation committee. Reprint requests should be sent to E.A.Krupinski, Department of Radiology, Radiology Research Division, University of Arizona Health Sciences Center, Tucson, AZ 85724. not consistently led to enhancement of detection performance. Some studies have reported an increase in the truepositive rate (e.g., for complex abnormalities other than tumors), but this gain has usually been offset by an accompanying increase in the false-positive rate. Even when observers have been provided with a cue that gives a complete physical and spatial description of the target abnormality, overall performance has not improved (Swensson et al., 1982).

These equivocal results run contrary to the results of many studies in psychology (Eriksen \& St. James, 1986; Eriksen \& Yeh, 1985; Krose \& Julesz, 1989; LaBerge, 1983; Treisman, 1964), in which cuing has consistently increased detection accuracy. Typically, however, accuracy performance is reported in terms of percent correct detections, rather than in terms of overall performance as measured by receiver operating characteristic (ROC) techniques, which account for false- as well as truepositive reports. Also, most laboratory search and detection studies have generally not been done with the types of multidimensional, camouflaged stimuli that are found in applied tasks such as the search for tumors in radiographs. The targets of search in laboratory tasks typically consist of overlearned letters or simple geometric shapes. Noise or distractors usually take the form of similar letters placed in the immediate vicinity of the target. These added distractors rarely interact with the target to camouflage it, and they typically do not generate many falsepositive reports.

In contrast, applied search tasks commonly consist of targets that are overlapped or camouflaged by background structures that have many similar features. Camouflaging has been shown to significantly impair detection performance in the search for military targets (King, Stanley, \& Burrows, 1984) and in the search for lesions in computer tomography (CT) images (Seltzer et al., 1991). Camouflage in $\mathrm{X}$-ray imaging is unique in that overlap- 
ping structures are translucent and contribute additively to gray-scale intensity rather than completely occluding one another as is typical in the physical world. Also, since the third dimension (depth) is represented by gray-scale intensity differences, $\mathrm{X}$-ray images contain few pictorial depth cues, making the task of disembedding and recognizing objects from the background even more difficult. Camouflaging and the similarity of target and background features contribute significantly to the high false-positive rates in radiology.

Additionally, images such as radiographs provide pictorial invariants (e.g., anatomic layout) that can help guide the search and detection of distinctive target-object features in a pictorial scene (Smets \& Stappers, 1990). Because the radiograph is searched for diagnostic purposes, the trained radiologist cannot help but interpret the anatomic scene that they have been trained to understand as they scan the image for abnormalities. Thus, searching and interpreting pictorial content are performed simultaneously. Pictorial interpretation is only minimally necessary when the search task is to detect a specific letter in a context of graphically similar letters.

Given the inconsistent findings from the cuing studies in radiology and the significant differences between laboratory and applied tasks and stimuli, it would seem that cuing holds little promise for aiding radiologists' detection performance. However, in a unique radiology study, Kundel, Nodine, and Krupinski (1990) used an eyeposition measure, visual dwell (gaze duration), to cue regions of interest (ROIs) in chest radiographs containing potential tumors. Visual dwell had previously been shown to predict the location of tumors in chest radiographs (Kundel, Nodine, \& Krupinski, 1989; Nodine, Carmody, \& Kundel, 1978). Of primary interest was the finding that approximately $65 \%$ of missed tumors are $f i x$ ated extensively with high-resolution central vision, yet are not recognized or reported. On average, gaze durations for regions containing missed tumors (false-negative decisions) were significantly longer ( $2.44 \mathrm{vs} .0 .51 \mathrm{sec}$ ) than those associated with tumor-free regions (truenegative decisions), and they were nearly as long as those associated with true-positive decisions $(2.76 \mathrm{sec})$. Thus, it seems as if tumors are missed not because of lack of foveal scrutinization, but rather because the tumorindicative features that attract scrutinizing are not recognized as being part of a target object.

In an attempt to improve radiologists' performance, Kundel et al. (1990) developed a method of computerassisted visual search (CAVS) that cued areas on chest images that had received prolonged dwell ( $>1,000 \mathrm{msec}$, as measured by eye-position recording) in an initial search of the image. The CAVS cuing consisted of highlighting image areas receiving prolonged dwell with a $5^{\circ}$ circle. The CAVS cuing resulted in an absolute increase (16\%) in tumor detection performance as measured by alterna"tive free-response operating characteristic (AFROC) analysis. The AFROC analysis is a variant of ROC analysis that is recommended (Chakraborty, 1989; Chakraborty \&
Winter, 1990) in experimental designs that are intended to maximize the number of decisions per observer by using multiple targets (thereby allowing multiple decisions) per image. The AFROC analysis also makes it possible to account for false-positive reports on target-containing images, which ROC analysis does not.

The increase in performance due to CAVS cuing was not due merely to a shift in criteria. Feedback cuing significantly increased the percentage of true-positive reports and decreased the percentage of false-positive reports in comparison with a second look without circle cues. The increase in true-positive reports was expected on the basis of the findings from the numerous cuing studies in psychology. The magnitude of the true-positive increase (20\%), however, was unexpected. What was even more unexpected, was the dramatic decrease in false-positive reports (by 27\%). Enhancement as large as this has not previously been reported in either radiology or psychology. Feedback cuing clearly resulted in a significant improvement in the discrimination of target from background noise in radiographic chest images.

A replication of this study (Krupinski, Nodine, \& Kundel, 1991) eliminated the use of CAVS (i.e., visual dwell) as the basis for cuing potential target sites and simply circled image locations likely to contain tumors. This type of experimental condition was more like those in which potential target locations have been cued either by providing a clinical history or by giving a complete spatial and physical description of the target, without any reference to what the radiologist may or may not have fixated during an initial search (see Berbaum et al., 1988; Berbaum et al., 1986; Carmody et al., 1980; Parker et al., 1982; Swensson et al., 1977, 1982). Circling alone without reference to eye-position data also resulted in a significant increase in performance $(21 \%)$, suggesting that cuing ROIs can be decoupled from the eye-position and dwell information and still facilitate detection.

In a follow-up study (Krupinski, Nodine, \& Kundel, 1993), different physical forms of cues (e.g., a dashedline circle) were compared; it was found that only a physically complete circle dramatically enhanced performance. For the exact same image locations and the same observers, less physically complete cues tended to increase the truepositive rates, but also resulted in more false-positive reports than did the complete circle. The pattern of an increase in both the true-positive and the false-positive rates is closer to the results of most of the other radiology cuing studies. This raises the question of why cuing by circling ROIs containing potential targets results in such a dramatic increase in observer performance when the other types of cuing seemingly do not.

The major goal of the present study was to determine why cuing by circling, in comparison with other means of cuing, substantially enhances detection performance in the task of searching for tumors in chest radiograph images. It is suggested that circle cuing physically isolates the ROI containing a potential tumor from the rest of the image, making the detection of camouflaged tumor fea- 
tures more probable. In addition, the circle may serve to isolate the ROI from distractors arising from the chest anatomy outside the boundary of the circle. This would facilitate the integration of potential target features detected within the ROI so that true tumors can be disembedded from background anatomy, which leads to recognition of these features as target objects.

In Experiment 1, we measured baseline detection performance without the aid of cuing, and then determined whether precuing alone (with two horizontal lines) was as effective at enhancing performance as precuing plus circling. In Experiment 2, we tested whether presentation time (tachistoscopic vs. extended viewing) was an important factor contributing to performance enhancement. To determine whether circle cuing influences visual scrutiny, in Experiment 3, we recorded eye position and measured fixation dispersion and precision with and without a circle present on the test image. In Experiment 4, we eliminated successive portions of the test image outside the ROI to determine whether the circle cue inhibits the processing of information outside the circled boundary of the ROI. In Experiment 5, we tested whether the circle cue reduces detection of distracting information outside the ROI by having observers make decisions about circled and uncircled tumors within and just outside the ROI.

\section{GENERAL METHOD}

\section{Image Display and Selection}

The test set consisted of 40 different chest images: 20 that were tumor-free, and 20 with a solitary simulated tumor ( $<50 \%$ detectability). Low-detectability tumors were used, since they are the type most likely to be missed and hence those most likely to benefit from cuing in an actual clinical setting. The chest images were digitally displayed at full size (on the average each image subtended $20^{\circ}$ of visual angle at a viewing distance of $70 \mathrm{~cm}$ ) on a 17-in., 525-line television monitor driven by a DeAnza Image Array Processor. (For complete image selection and digitization procedures see Kundel et al., 1990.) The tumors had a Gaussian edge profile and their diameters ranged from 0.8 to $2.0 \mathrm{~cm}$, which at a viewing distance of $70 \mathrm{~cm}$ subtended $0.65^{\circ}-1.64^{\circ}$ of visual angle. The set of 40 test images was replicated to produce a second set of images. A $5^{\circ}$-diameter, 2-pixel-wide black circle was placed on each of the images in one set (cue condition). No circles were added to the second set (no-cue condition).

For the 20 tumor-containing images in the cue condition, a circle cue was placed around each tumor region and randomly offset so that the tumors did not always appear in the center of the circle. The tumors had to lie entirely within the circle. A description of the tumor locations based on their polar coordinates with respect to the center of the circle indicated that approximately two thirds of the tumors were about halfway between the center and the edge of the circle; the remaining one third were closer to either the center or the edge. None of the tumors appeared directly in the center of the circle. An analysis of the Kundel et al. (1990) data indicated very little correlation $(r=.17)$ between the tumor location within the circle and the probability of the tumor's being reported (i.e., tumors closer to the circle edge were just as likely to be detected as those towards the center). For tumor-free images, each circle was placed on an anatomically matched region corresponding to each circled region in the tumor-containing images.

To fully counterbalance the design, the test images were assigned randomly to blocks of 10 images, each having 5 tumor-free and 5 tumor-containing images. Each block contained either cue or no- cue images, but not both. Presentation of blocks of images in the various experimental conditions was counterbalanced for cue presence.

\section{Procedure}

All of the experiments presented below followed the same procedure unless explicitly stated otherwise. In half-hour sessions separated on the average by 2.5 weeks, observers (radiology residents having an average of 2.5 years of experience at reading radiographs) viewed the blocks of images, each in a different random order. Each image was preceded by a precue image. The precue image background was an unstructured gray field matched in intensity to the average intensity value of the 40 chest images $\left(5.0 \mathrm{~cd} / \mathrm{m}^{2}\right)$. The precue consisted of two black lines each $1 \mathrm{~cm}$ in length horizontally tangent to the top and bottom of the $5^{\circ}$ region on the test image. Each precue designated the same location and area that the cue designated. For the no-cue condition, the precue appeared in the same location as that in which a cue might otherwise appear, so that direct comparisons could be made between conditions. A complete circle was not used as a precue, to avoid the possibility of an afterimage on the test image in the no-cue condition.

Figure 1 shows the display sequence. The observers were seated $70 \mathrm{~cm}$ from the display and used a chinrest/head restraint to maintain a constant viewing position throughout the experiment. The average room illumination was set at $5 \mathrm{~cd} / \mathrm{m}^{2}$, making it equivalent to the average luminance level of the display. The observers were instructed to fixate the precued location, and when they were ready, to press a joystick button to present a test image. The time required to switch images was $33 \mathrm{msec}$. The DeAnza Image Array Processor has three image channels and was programmed to function as a tachistoscope. The first channel held the precue image, the second held the test image, and the third held the mask image. Each test image was displayed for $200 \mathrm{msec}$, which was short enough to make an eye movement unlikely (Hock, 1984; Yarbus, 1967). The test image was then removed and replaced by a randomized noise pattern for $200 \mathrm{msec}$ to terminate visual processing of the image. A gray field, intensity matched to the precue field, then appeared while the next image was being loaded.

After the observer had seen the three-image sequence (precue, test, mask), he/she reported whether the indicated location was tumor free or tumor containing and rated his/her decision confidence on a five-level rating scale $(5=$ tumor, definite; $4=$ tumor, probable; 3 = suspicious; 2 = no-tumor, probable $11=$ no-tumor, definite). Each observer viewed a set of 20 practice images before viewing any test images, in order to become acquainted with the test procedure, with viewing the images tachistoscopically, and with the type of target tumor used in the test images.

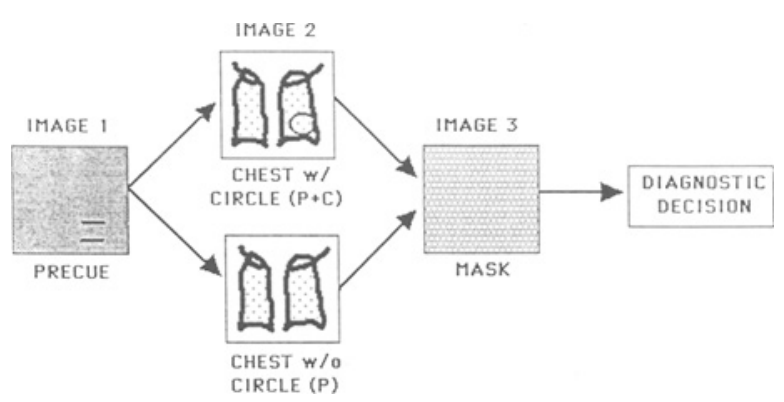

Figure 1. General design of the tachistoscopic experiments. Observers are precued to a specific location in the display field by initially presenting a gray-field image with two horizontal bars that specify a $5^{\circ}$ region. The stimulus chest image is then presented tachistoscopically for $200 \mathrm{msec}$, with or without a circle cue at the precued location. A random noise mask follows presentation of the chest image, and a diagnostic decision about the specified location is made. 
Performance was measured by ROC area under the curve (Az) values and the differences between $\mathrm{Az}$ values for each condition were analyzed in a repeated measures analysis of variance (ANOVA) and appropriate post hoc tests. The ROC Az values were used because $A z$ is a criterion-free, parameter-free, distribution-independent index of performance accuracy (Massof \& Emmel, 1987; Swets, 1979 ) that is typically used in studies measuring medical diagnostic performance.

\section{EXPERIMENT 1}

\section{Method}

In Experiment 1, we measured baseline performance of 5 radiology residents in detecting very subtle tumors in chest $\mathrm{X}$-ray images and determined whether precuing was as effective in enhancing detection performance as precuing followed by cuing (circling) the ROI on the image. In the precue-only (P) condition, presentation of each chest image was preceded by the precue image. In the precue-pluscue $(P+C)$ condition, the precue image was followed by the chest image with the $5^{\circ}$ circle cue delineating the ROI. The $\mathrm{P}$ and $\mathrm{P}+\mathrm{C}$ images were presented tachistoscopically $(200 \mathrm{msec})$. In the free search (FS) condition, the observers viewed the test images for $15 \mathrm{sec}$, without any precuing or cuing (baseline performance). Tachistoscopic presentation was not used in the FS condition, because previous studies (Gale \& Worthington, 1984; Kundel \& Nodine, 1975 ) have shown that subtle tumors have very low detection rates when chest $\mathrm{X}$-ray images are presented tachistoscopically to observers fixating the center of the display (i.e., without precuing).

\section{Results}

The mean Az value for FS was 0.502 ( $S E=.049$, where 0.50 is chance); for $\mathrm{P}$, it was $0.608(S E=.018)$; and for $\mathrm{P}+\mathrm{C}, 0.767(S E=.019)$. A repeated measures ANOVA of individual Az values revealed a significant treatment effect $[F(2,8)=17.84, p<.001]$ and the post hoc tests (Fisher protected least squares difference) indicated that each of the three conditions differed significantly from the others.

At least for images such as those used here, in which detection performance is chance in the absence of precuing, the results indicate that although a precue leads to an increase in Az performance over free search, the added presence of a circle cue physically delineating the ROI on the test image leads to an even greater significant increase in $\mathrm{Az}$ performance over precuing only.

\section{EXPERIMENT 2}

\section{Method}

Were the results of Experiment 1 produced by the tachistoscopic presentations of the images? A second group of 4 radiology residents viewed the chest images in both a 200 -msec tachistoscopic (precluding shifts in eye position) presentation and a 2,000-msec (allowing for eye-position shifts) presentation. Two of the observers viewed randomized blocks of $P$ and $P+C$ images in the 200 -msec condition during an initial session, and then in the 2,000 -msec condition in a second session ( 3 weeks later). The other 2 observers viewed the same conditions in the opposite order, to counterbalance for practice effects. Thus, each observer saw the images in each of four conditions: $P, 200 \mathrm{msec} ; \mathrm{P}+\mathrm{C}, 200 \mathrm{msec} ; \mathrm{P}, 2,000 \mathrm{msec}$; and $\mathrm{P}+\mathrm{C}, 2,000 \mathrm{msec}$.

\section{Results}

The results of Experiment 2 indicate that the previous findings were not produced by brief presentation of the images. The average difference in $\mathrm{Az}$ values between the $200-$ and 2,000-msec conditions was $0.032(S E=.023)$ and $0.033(S E=.027)$ for the $\mathrm{P}$ and $\mathrm{P}+\mathrm{C}$ conditions, respectively.

Analysis of the differences with a two-factor repeated measures ANOVA indicated that the main effect of presentation time ( 200 vs. $2,000 \mathrm{msec})$ was not statistically significant $[F(1,3)=1.88$, n.s. $]$. The $95 \%$ confidence intervals also indicate that the two means overlap (Az $200 \mathrm{msec}=.628, S E=.039 ; \mathrm{Az} 2,000 \mathrm{msec}=.660$, $S E=.040)$. The main effect of condition ( $\mathrm{P}$ vs. $\mathrm{P}+\mathrm{C})$ was highly significant $[F(1,3)=497.39, p<.0002]$, but the time $\times$ condition interaction was not. Post hoc Scheffé $F$ tests indicated that neither $\mathrm{P}$ nor $\mathrm{P}+\mathrm{C} 200$ differed from 2,000 , but the $P+C$ conditions were significantly higher than either $\mathrm{P}$ condition $(p<.0001$ in each case).

In both the $\mathrm{P}$ and the $\mathrm{P}+\mathrm{C} 2,000$-msec conditions, the observers were precued to a potential target location and given sufficient time to fully engage focused processing resources to search the ROI, yet the observers still performed significantly better (i.e., true-positive increase and false-positive decrease) when a circle cue physically delineated the ROI on the test image than when it did not.

\section{EXPERIMENT 3}

How does the presence as opposed to the absence of the circular boundary on the chest image affect radiologists' eye movements at the precued area? Numerous studies have demonstrated that various eye-position parameters (e.g., gaze duration, saccade length, fixation density) are reliable measures of information-processing strategies and attention allocation (Just \& Carpenter, 1988; Kundel et al., 1989). In Experiment 3, we utilized two of these eyeposition parameters (fixation dispersion and precision) to determine whether circle cuing influences visual scrutiny.

\section{Method}

Twenty of the tumor-containing and 5 of the tumor-free chest images from Experiment 1 were used. Since the main concern was with analyzing eye-position parameters with respect to sampling of tumors with and without a circle cue present rather than observer performance, fewer tumor-free images were required.

Four radiology residents who had not participated previously viewed the $\mathrm{P}$ and $\mathrm{P}+\mathrm{C}$ images for $15 \mathrm{sec}$ while their eye position was recorded. The observers could terminate search before $15 \mathrm{sec}$ by pushing a joystick button, which removed the image from view. On the average, search was terminated after $5 \mathrm{sec}$. Eye position was recorded (Eye-Trac Model 210; Applied Science Laboratories, Waltham, MA) according to a limbus-reflection technique (see Nodine, Kundel, Toto, \& Krupinski, 1992, for complete details of the eye-position recording and analysis procedures).

Two parameters of the eye-position data were analyzed. The first was the dispersion of fixations generated during search calculated for every $500 \mathrm{msec}$ of search. Dispersion was measured by determining the mean location of the fixations generated during each $500 \mathrm{msec}$ of search, and by calculating the standard deviation or the 
degree to which fixations were spread out over the ROI. Since the observers viewed each image both with and without a circle, dispersion could be compared for a given observer on a given image with and without a circle for each 500-msec interval. The second parameter was precision calculated as the mean distance between each of the observer-generated fixations and the center of the tumor for a given observer on a given image with and without a circle for each 500 -msec interval.

\section{Results}

The mean dispersion of eye fixations was $0.89^{\circ}(S E=$ $\left..013^{\circ}\right)$ when a circle was present on the chest image, and $1.13^{\circ}\left(S E=.014^{\circ}\right)$ when the circle was absent. The mean dispersion was $0.24^{\circ}$ smaller with than without the circle cue, which, when analyzed in a one-factor repeated measures ANOVA, was found to be statistically significant $[F(1,3)=14.43, p<.05]$. Thus, when the circle was present, the fixations covered $18 \%$ of the $5^{\circ} \mathrm{ROI}$, and when the circle was absent, they covered $24 \%$ of the $5^{\circ} \mathrm{ROI}$.

To analyze precision, only the tumor-containing images were included in the analysis. Precision was $1.75^{\circ}$ ( $S E=$ $\left..01^{\circ}\right)$ when the circle was present, and $1.95^{\circ}\left(S E=.01^{\circ}\right)$ when the circle was absent. Additionally, $15 \%$ of the fixations fell directly on the tumor when the circle was present, while only $8 \%$ fell directly on the tumor when the circle was absent. The difference between the two conditions was $0.21^{\circ}$, which was marginally significant $[F(1,3)=9.11, p<.06]$ when tested in a one-factor repeated measures ANOVA. For both precision and dispersion, the difference between the circling and nocircling images occurred for both true-positive and falsenegative decisions. The degree of precision and dispersion was not a function of tumor location within the circle.

\section{EXPERIMENT 4}

Does the circle cue inhibit processing of information outside the circled boundary of the ROI? If so, the physical elimination of image content outside the ROI should have little effect on performance when the circle cue is present; but when it is absent, the elimination of image content should improve performance.

\section{Method}

Ten radiology residents who had not previously participated viewed the chest images in each of six counterbalanced conditions. Image content was eliminated by removing either half or three quarters of the image pixels outside of a concentric ring surrounding the $5^{\circ}$ ROI. The removed pixels were replaced by a gray field matched to the average intensity of the chest images. After these operations were performed, the chest images were randomized and

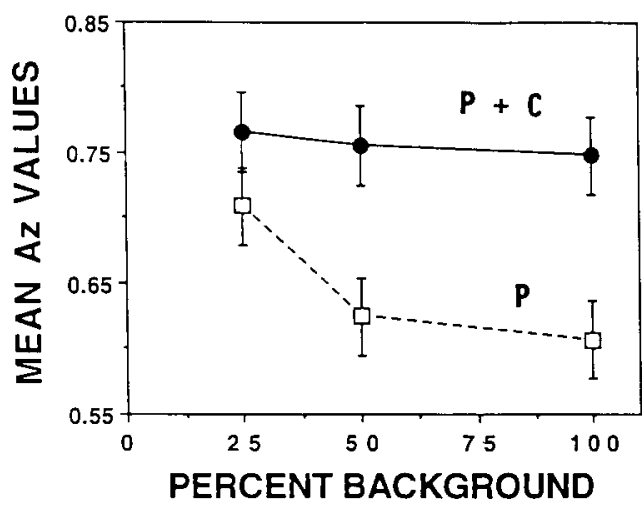

Figure 2. $P$ (precue-only condition; open squares) and $P+C$ (precue + cue condition; closed circles) mean Az values for the $25 \%$, $50 \%$, and $100 \%$ background conditions in Experiment 3 .

presented tachistoscopically in the $\mathrm{P}$ and $\mathrm{P}+\mathrm{C}$ conditions with three percentages of image content outside the ROI present: $25 \%, 50 \%$, and $100 \%$.

\section{Results}

Figure 2 shows the mean $\mathrm{Az}$ values for the $\mathrm{P}$ and $\mathrm{P}+\mathrm{C}$ $25 \%, 50 \%$, and $100 \%$ conditions. The $\mathrm{Az}$ values were tested for significant differences in a two-factor repeated measures ANOVA. The main effect of condition ( $P$ vs. $\mathrm{P}+\mathrm{C})$ was statistically significant $[F(1,9)=51.47, p<$ $.0001]$, as was the main effect of background $(25 \%, 50 \%$, $100 \%)[F(2,18)=20.01, p<.0001]$. The interaction term, condition $\times$ background, was statistically significant $[F(2,18)=39.56, p<.001]$. Post hoc Scheffé $F$ tests indicated that none of the $\mathrm{P}+\mathrm{C}$ background conditions was significantly different from another. In the $P$ condition, $100 \%$ background was not significantly different from $50 \%$, but $100 \%$ versus $25 \%$ and $50 \%$ versus $25 \%$ were both significantly different $(p<.01)$. The mean differences between $\mathrm{P}$ and $\mathrm{P}+\mathrm{C}$ conditions for each percent background $(25 \%, 50 \%, 100 \%)$ were all statistically significant.

The overall true- and false-positive fractions remained relatively constant for each change in image content in the $\mathrm{P}+\mathrm{C}$ condition (see Table 1). The true-positive fraction was consistently higher in the $\mathrm{P}+\mathrm{C}$ than in the $\mathrm{P}$ condition for each percent background by approximately $10 \%$. In the $\mathrm{P}$ condition, the true-positive fractions also remained relatively constant across backgrounds, but the false-positive fractions progressively decreased until they approximated $\mathrm{P}+\mathrm{C}$ false-positive fractions. As can be

Table I

Percentage of Positive Decisions per Decision Category for $P$ and $\mathbf{P}+\mathbf{C}$ Conditions for $\mathbf{2 5 \%}, \mathbf{5 0} \%$, and $100 \%$ Backgrounds in Experiment 3

\begin{tabular}{ccccccccc}
\hline & \multicolumn{3}{c}{ P Background Present } & & \multicolumn{3}{c}{ P +C Background Present } \\
\cline { 2 - 4 } \cline { 7 - 8 } Decision & $25 \%$ & $50 \%$ & $\mathbf{1 0 0 \%}$ & & $25 \%$ & $50 \%$ & $100 \%$ \\
\hline True positive & 61 & 57 & 59 & & 70 & 70 & 72 \\
False positive & 29 & 38 & 43 & & 32 & 33 & 30 \\
\hline
\end{tabular}

Note-P, precue only; $P+C$, precue plus cue. 
seen in Figure 2, the decrease in the false-positive fraction represents an increase in overall performance in the $25 \%$ background condition in comparison with the $50 \%$ and $100 \%$ conditions.

Eliminating image content outside the ROI significantly enhanced performance in the $\mathrm{P}$ condition until it approximated performance in the $\mathrm{P}+\mathrm{C}$ condition. Eliminating the background in the $\mathrm{P}+\mathrm{C}$ condition did not significantly affect performance.

\section{EXPERIMENT 5}

The results of Experiment 4 suggest that circling perceptually isolates the ROI from the rest of the image. The purpose of this perceptual isolation could be overridden by an instruction to report on the presence or absence of tumor targets both inside and immediately outside the circle.

\section{Method}

On half of the tumor-containing images $(n=10)$ and half of the tumor-free images $(n=10)$, a distractor tumor was placed outside but within $2.5^{\circ}$ of the boundary of the $5^{\circ} \mathrm{ROI}$. There were thus two zones of interest: inside and outside the ROI (see Figure 3). The outside zone was not delineated in any physical manner, but prior to each testing session, the observers were shown a photograph similar to the one in Figure 3, and the extent of the two zones was also delineated on a sample image displayed on the viewing monitor. The distractor tumors were of the same size and conspicuity as were the primary tumors.

Four radiology residents who had not participated in any previous studies served as the observers. The images were counterbalanced for $\mathrm{P}$ and $\mathrm{P}+\mathrm{C}$ conditions and presented tachistoscopically in a random block design. Within any block of images, there were images both with and without distractor tumors present. The observers were informed that for both the inside and the outside zones, the target and nontarget alternatives were equiprobable and independent. The observers were instructed to make two decisions per image: one about the presence or absence of a tumor inside the ROI (inside decision) and a second about the presence or absence of a tumor in the outer zone (outside decision). We did not

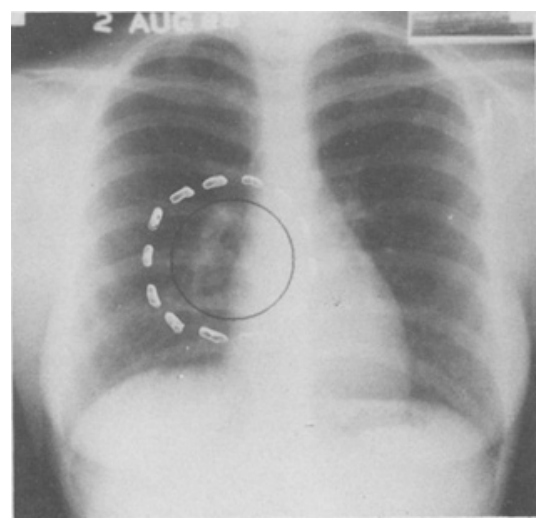

Figure 3. A typical example of a chest image used in Experiment 5. The area within the solid-line black circle represents the inside zone of interest and the area between this line and the dotted-line white circle represents the outside zone of interest. The dotted-line white circle did not appear on the test images.
Table 2

Proportion of True-Positive and False-Positive Reports for the Inside and Outside Zones in Experiment 5

\begin{tabular}{cccccc}
\hline & \multicolumn{2}{c}{$\mathrm{P}$} & & \multicolumn{2}{c}{$\mathrm{P}+\mathrm{C}$} \\
\cline { 2 - 3 } \cline { 5 - 6 } Decision & Inside & Outside & & Inside & Outside \\
\hline True positive & .59 & .35 & & .81 & .18 \\
False positive & .44 & .16 & & .36 & .15 \\
\hline
\end{tabular}

Note-P, precue only; $P+C$, precue plus cue

counterbalance order of reporting inside and outside targets, be cause we were specifically concerned with the question of whether the presence of the circle cue would reduce the detection of distracting information (even if the distractor was a target) outside its boundaries, given that search had been directed to the ROI inside the circle.

\section{Results}

Performance in the $\mathrm{P}$ and $\mathrm{P}+\mathrm{C}$ conditions was compared by looking at the proportion of true- and falsepositive decisions in the inside and outside zones of interest for the two conditions (see Table 2). For the inside zone, there were $22 \%$ more true-positive and $8 \%$ fewer false-positive decisions in the $\mathrm{P}+\mathrm{C}$ than in the $\mathrm{P}$ condition. Using $z$ tests for proportions to test for significant differences, the proportion of true positives was significantly different $(z=3.10, p<.01)$, whereas the difference in false positives was not. Conversely, for the outside zone, there were $17 \%$ more true-positive and $1 \%$ more false-positive decisions in the $\mathrm{P}$ than in the $\mathrm{P}+\mathrm{C}$ condition. The proportion of true positives was significantly different $(z=2.35, p<.01)$, whereas the proportion of false positives was not significantly different. These differences in detection rates were not a function of whether one or two tumors were reported. In both the $\mathrm{P}$ and $\mathrm{P}+\mathrm{C}$ conditions, the rate of reporting tumors in the outside zone was the same, whether or not the observer had reported a tumor in the inside zone. A comparison of the true- and false-positive rates for the $\mathbf{P}$ and $\mathrm{P}+\mathrm{C}$ conditions in this experiment and those for the $100 \%$ background condition in Experiment 3 (Tables 1 and 2) supports this.

With respect to overall performance (ROC Az) for the inside zone of interest, mean $\mathrm{Az}$ performance in the $\mathrm{P}+\mathrm{C}$ condition $(.807, S E=.007)$ was significantly higher than performance in the $\mathrm{P}$ condition $(.611, S E=.018)$ $[F(1,3)=71.52, p<.03]$. For the outside zone of interest, performance in the $\mathrm{P}$ condition (.621, $S E=.007$ ) was higher than performance in the $P+C$ condition (.560, $S E=.012$ ), but the difference was not statistically significant $[F(1,3)=4.76$, n.s. $]$.

The results support the suggestion that circling isolates the ROI from distractors outside the boundary (even if the distractor is a target). When a circle was present, fewer tumor targets in the outside zone were detected than when the circle was absent. This finding was independent of whether or not a target was reported (with or without a circle) in the inside zone. 


\section{GENERAL DISCUSSION}

The results of the five experiments presented here have demonstrated that detection performance for tumors embedded in chest $\mathrm{X}$-ray images is significantly enhanced when precuing of potential target locations is followed by a circle cue on the test image that physically bounds the ROI in comparison with precuing only or search without precuing (Experiment 1). Even extending search time so that the observers were given adequate time to more accurately localize the potential target site and engage in a more extensive search of the ROI did not significantly enhance performance in the $\mathrm{P}$ condition (Experiment 2).

Alone, the precue focuses attention to a specified image location, and although it enhances tumor detection somewhat, it does not seem to facilitate the rejection of noise, as evidenced by the high false-positive rates in the various $\mathbf{P}$ conditions as opposed to the $\mathrm{P}+\mathrm{C}$ conditions. We believe that the circle cue significantly enhances performance beyond precue-only performance because it physically delineates the ROI on the image, limiting attention and search by compelling the visual system to ignore pictorial scenery outside the cue boundary. This outside scenery contains features that distract attention and interfere with the discrimination of target and noise. The suggestion that the circle cue facilitates signal detection/recognition and rejection of noise is supported by the consistently higher true-positive and lower false-positive rates in the $\mathrm{P}+\mathrm{C}$ than in the $\mathrm{P}$ condition.

Circle cuing increased the efficiency of visual sampling within the boundary of the ROI, increasing the likelihood that target features were detected (Experiment 3). The circle exerted a profound influence on the way scanning takes place within the ROI. The circle cue tightened the dispersion of eye fixations and increased the precision and percentage of fixations that fell directly on the tumors. Each of these factors increases the likelihood of the eye's sampling the tumor with foveal vision, even though in both conditions the precue localized attention and the axis of gaze to the spatial location and extent of the ROI. In terms of information-processing theory, the increased hit rate in sampling the target could mean (1) that feature detection and disembedding from the background was more likely, and (2) that there was more target-relevant scrutiny leading to effective feature integration.

The results of Experiment 3 specifically addressed the question of how the presence of the circle cue could be enhancing the detection of the tumors. Experiments 4 and 5 also provided information with respect to this question, as well as the question of why the circle cue tends to significantly decrease the false-positive rate when other types of cues do not seem to be able to accomplish this. Thus, it is interesting to note that in Experiment 4 the increase in $P$ performance as image content outside the ROI was removed was accompanied by a significant decrease in false-positive decisions (along with increases in truepositive decisions). In the $\mathrm{P}+\mathrm{C}$ condition, the false- positive rate remained fairly constant (and lower than in the $\mathbf{P}$ condition) in each background removal condition. This result is what one might expect if, without a circle to bound the ROI and indicate what to ignore, distracting nontarget features in proximity to the ROI are being confounded with true target features and called falsely positive. As the amount of image content outside the ROI decreases, it is easier for the visual/attention system to ignore the background information even without a circle present, improving performance.

The results of Experiment 5 also clearly support the interpretation that cuing by circling the ROI reduces the interfering effects of distractors outside its boundaries by telling the visual system to ignore this information. Even when explicit instructions were given to divide attention between inside and outside the circle boundary, the percentage of true-positive decisions in the $\mathrm{P}+\mathrm{C}$ condition indicates that this was very difficult to accomplish. Without a circle cue present, significantly more of the outside tumors were detected than with a circle present. It should be noted that in both the $\mathrm{P}+\mathrm{C}$ and $\mathrm{P}$ conditions, detection was significantly lower for the outside than for the inside tumors, but as was noted previously, this was independent of whether or not a tumor had been reported in the inside zone. The lower detection rate probably had more to do with the fact that peripheral vision was required to detect the outside tumors (Nodine et al., 1992).

Such explicit differences between precuing and cuing attention processes have not previously been reported, in part because such complex stimuli with subtle, camouflaged targets have not generally been studied. However, these types of displays are frequently encountered in applied search and detection tasks not only in medical radiography, but also in areas such as photointerpretation (e.g., military search and detection of targets; industrial X-ray scanning of machine parts). In these tasks, false-positive reports become a significant problem because the observer must interpret the pictorial content of the scene while searching for a target. It is perhaps because of this additional task requirement in applied tasks such as radiology that cuing by circling enhances detection performance.

\section{REFERENCES}

Bassett, L. W., Manjikian, V., \& Gold, R. H. (1990). Mammography and breast cancer screening. Surgical Clinics of North America, 70, 775-800.

Berbaum, K. S., Franken, E. A., Dorfman, D. D., \& Barloon, T. J. (1988). Influence of clinical history upon detection of tumors and other lesions. Investigative Radiology, 23, 48-55.

Berbaum, K. S., Franken, E. A., Dorfman, D. D., Barloon, T., Ell, S. R., Lu, C. H., SMITH, W., \& ABU-YousefF, M. M. (1986). Tentative diagnoses facilitate detection of diverse lesions in chest radiographs. Investigative Radiology, 21, 532-539.

Carmody, D. P., Nodine, C. F., \& Kundel, H. L. (1980). Global and segmented search for lung tumors of different edge gradients. Investigative Radiology, 15, 224-233.

Chakraborty, D. P. (1989). Maximum likelihood analysis of freeresponse receiver operating characteristic (FROC) data. Medical Physics, 16, 561-568. 
Chakraborty, D. P., \& Winter, L. H. L. (1990). Free-response methodology: Alternate analysis and a new observer-performance experiment. Radiology, 174, 873-881.

ERIKSEN, C. W., \& ST. JAmes, J. D. (1986). Visual attention within and around the field of focal attention: A zoom lens model. Perception \& Psychophysics, 40, 225-240.

ERIKSEN, C. W., \& YEH, Y. (1985). Allocation of attention in the visual field. Journal of Experimental Psychology: Human Perception \& Performance, 11, 583-597.

Gale, A. G., \& Worthington, B. S. (1984). Image viewing habits and the false diagnosis. Diagnostic Imaging, 11, 130-133.

Hock, H. S. (1984). Christina's world: Imaginary perspectives and the encoding of spatial alignment relations. In W. R. Crozier \& A. T. Chapman (Eds.), Cognitive processes in the perception of ant (pp. 167188). New York: Elsevier.

Just, M. A., \& CARPENTER, P. A. (1988). Reading and spatial cognition: Reflections from eye fixations. In G. Luer \& J. Shallo-Hoffman (Eds.), Eye movement research: Physiological \& psychological aspects (pp. 193-213). New York: Hogrefe.

King, M., Stanley, G. V., \& Burrows, G. D. (1984). Visual search in camouflage detection. Human Factors, 26, 223-234.

Krose, B. J. A., JULESZ, B. (1989). The control and speed of shifts of attention. Vision Research, 29, 1607-1619.

Krupinski, E. A., Nodine, C. F., \& KUndel, H. L. (1991, September). Highlighting long gaze durations enhances observer performance by reducing the influence of distracting background information. Paper presented at the 6th European Conference on Eye Movements, Leuven, Belgium.

Krupinski, E. A., Nodine, C. F., \& Kundel, H. L. (1993). A perceptually-based method for enhancing pulmonary nodule recognition. Investigative Radiology, 28, 289-294.

KUNDEL, H. L., \& NoDiNE, C. F. (1975). Interpreting chest radiographs without visual search. Radiology, 116, 527-532.

Kundel, H. L., Nodine, C. F., \& Krupinski, E. A. (1989). Searching for lung nodules: Visual dwell indicates locations of false positive and false negative decisions. Investigative Radiology, 24, 472-478.

Kundel, H. L., Nodine, C. F., \& Krupinsk, E. A. (1990). Computerdisplayed eye position as a visual aid to pulmonary tumor interpretation. Investigative Radiology, 25, 890-896.

LABERGE, D. (1983). Spatial extent of attention to letters in words. Journal of Experimental Psychology: Human Perception \& Performance, 9, 371-379.
Massof, R. W., \& Emmel, T. C. (1987). Criterion-free parameter-free distribution-independent index of diagnostic test performance. Applied Optics, 26, 1395-1408.

Muhm, J. R., Miller, W. E., Fontan, R. S., Sanderson, D. R., \& UHLENHOPP, M. A. (1983). Lung cancer detection during a screening program using four-month chest radiographs. Radiology, 148, 609-615.

Nodine, C. F., Carmody, D. P., \& Kundel, K. L. (1978). Searching for NINA. In J. W. Senders, D. F. Fisher, \& R. A. Monty (Eds.), Eye movements and higher psychological functions (pp. 317-328). Hillsdale, NJ: Erlbaum.

Nodine, C. F., Kundel, H. L., Toto, L. C., \& Krupinski, E. A (1992). Recording and analyzing eye-position data using a microcomputer workstation. Behavior Research Methods, Instruments, \& Computers, 24, 475-485.

Parker, T. W., Kelsey, C. A., Moseley, R. D., Mettler, F. A., GARCIA, J. F., \& Briscoe, D. E. (1982). Directed versus free search for tumors in chest radiographs. Investigative Radiology, 17, 152-155.

Seltzer, S. E., Swensson, R. G., Nawfel, R. D., Lentini, J. F., KAZDA, I., \& JUDY, P. (1991). Visualization and detection-localization on computed tomographic images. Investigative Radiology, 26, 285-294.

SMETS, G. J. F., \& Stappers, P. J. (1990). Do invariants or features determine the conspicuity of forms? In D. Brogan (Ed.), Visual search (pp. 29-36). New York: Taylor \& Francis.

Swensson, R. G., Hessel, S. J., Herman, P. G. (1977). Omissions in radiology: Faulty search or stringent reporting criteria? Radiology, 123, 563-567.

Swensson, R. G., Hessel, S. J., Herman, P. G. (1982). Radiographic interpretation with and without search: Visual search aids the recognition of chest pathology. Investigative Radiology, 17, 145-151.

SWETS, J. A. (1979). ROC analysis applied to the evaluation of medical imaging techniques. Investigative Radiology, 14, 109-121.

Treisman, A. (1964). Verbal cues, language, and meaning in selective attention. American Journal of Psychology, 77, 206-219.

YARBus, A. L. (1967). Eye movements and vision. New York: Plenum.

(Manuscript received April 28, 1992; revision accepted for publication November 5,1992 .) 